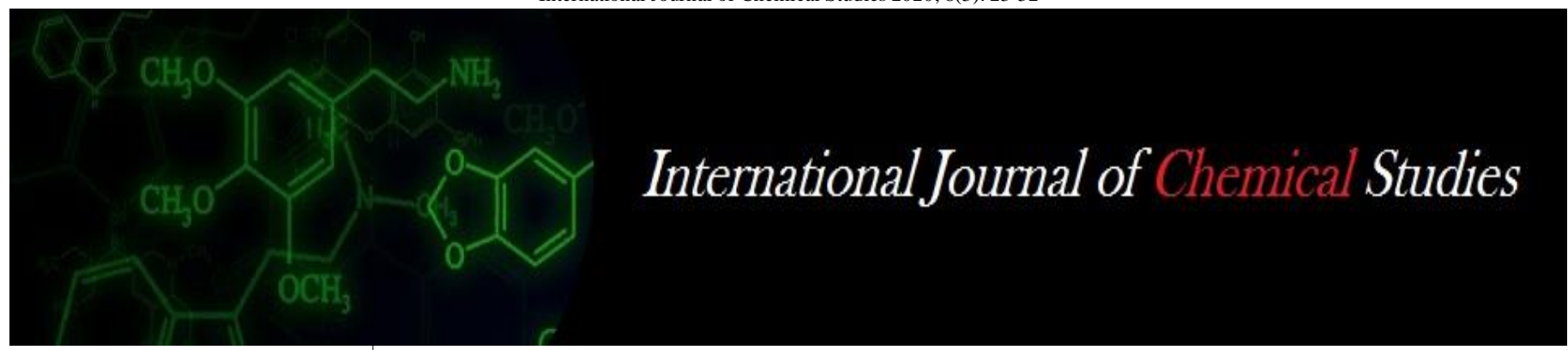

P-ISSN: 2349-8528

E-ISSN: 2321-4902

www.chemijournal.com

IJCS 2020; 8(3): 23-32

(C) 2020 IJCS

Received: 16-03-2020

Accepted: 18-04-2020

PB Gujarathi

Department of Chemistry

Shri Shivaji College kandhar,

Dist. Nanded, Maharashtra,

India
Corresponding Author: PB Gujarathi

Department of Chemistry Shri Shivaji College kandhar, Dist. Nanded, Maharashtra, India

\section{Review on synthetic advances in porphyrins and metalloporphyrins}

\section{PB Gujarathi}

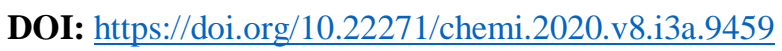

\begin{abstract}
Due to extensive applications of porphyrin-based materials today, this paper aims to present review on the synthesis of the first and principal methodologies along with recent synthetic developments in this macrocyclic molecule.
\end{abstract}

Keywords: Porphyrin, metalloporphyrin, microwave irradiation, organometallic synthesis

\section{Introduction}

Porphyrin and its derivatives are among the most widely distributed and important cofactors found in nature and are crucial regulatory effectors in many biochemical processes. Additionally, efforts to understand the physicochemical characterstics, conformational flexibility and aromaticity of porphyrin and its derivatives has led to the development of their applications in vast areas such as photodynamic therapy (PDT), ${ }^{[1]}$ redox catalysis, metal coordination, sensors, nonlinear optics (NLO) and nanomaterials ${ }^{[2]}$ etc.

Initial synthetic investigations in this area were initiated by groups of Rothemund, ${ }^{[3]} \mathrm{Adler}^{[4]}$ and Lindsey ${ }^{[5]}$ etc. The Adler methodology though low yielding, is prized for its simplicity and applicability for the large scale preparation of meso-aryl porphyrins. The in situ formed porphyrinogen intermediate which get oxidized leading to the formation of. The Lindsey's group subsequently developed higher concentration conditions (0.1-0. 3 moll-1) that were slightly lower yielding than before, ${ }^{[4]}$ but more practical for larger scale preparations. ${ }^{[6,7]}$ More recently, it has also been found that the addition of salts, such as sodium choride, during the condensation reaction of pyrrole and aldehyde increases the yield ${ }^{[8]}$.

Different variants of the Rothemund ${ }^{[3]}$ or Lindsey's ${ }^{[8]}$ procedure employ hydroiodic acid, hydrochloric acid, p-toluene sulfonic acid (p-TsOH) ${ }^{[9]}$, perchloric acid, trichloroacetic acid, trifluoroacetic acid, montmorillonite clay, cation-exchange resins, high 4 valent transition metals, metal triflate as catalyst and/or oxidants and are synthetically useful. A solvent as well as catalyst-free preparation of meso-substituted porphyrins through the reaction of pyrrole and aldehydes, together in the gas phase $\left(>200^{\circ} \mathrm{C}\right)$, using oxygen as oxidant, has been reported to furnish tetraphenyl porphyrin in $23 \%$ yield. Earlier methodologies employing an aldehyde and pyrrole suffered in that there was no provision of bringing variations of substituents at the four meso-positions, a limiting factor to form large covalently bonded arrays. Further, if a mixture of two or more different aldehydes is employed in the above synthesis, a statistical mixture of products is obtained and require extensive chromatography for isolation of the desired porphyrin, generally formed in low yield by virtue of the statistical outcome of the reaction.

Alternative approaches for synthesizing substituted porphyrins have been described in which pre-functionalized dipyrromethane derivatives are condensed with similar diformyl dipyrromethanes through a " $2+2$ " condensation approach to form meso-tetraarylporphyrins. Such condensations ${ }^{[9]}$ are usually catalyzed by acids and the intermediate porphyrinogens are oxidized by air to obtain the desired porphyrins. In a similar " $3+1$ " synthetic approach, tripyrranes are condensed with 2, 5- diformylpyrroles to form etioporphyrin. Another modified " $2+2$ " condensation approach involves the acid catalyzed condensation of $\alpha$-free dipyrromethanes with aldehydes to form porphyrinogens, which are then chemically oxidized to obtain porphyrins. This methodology is considerably more versatile for array formation, and is frequently higher yielding and produces more soluble products as well as allows better 
control over substitution at the meso-positions.

In view of the extensive literature on the development of synthetic methodologies in porphyrin chemistry as well as applications in a variety of areas, a number of comprehensive review articles have been published ${ }^{[10]}$ and their discussion is beyond the scope of the present investigation.

Each method has its own utilities and limitations, herein we covers the earlier basic methodologies and some recent greener methodologies such as organometallics, clay catalyzed, microwave assisted, ionic liquid mediated reactions have been discussed.

\section{Synthesis of functionalized porphyrins from non- porphyrin precursors}

Meso-substituted unsymmetrical porphyrins are key structural components found in a wide range of model systems in biomimetic and material chemistry. Over the past years, much effort has been extended on development of synthetic approaches to octaalkylporphyrins and natural porphyrins related to heam and chlorophyll. Procedures have advanced symmetrically through monopyrrole tetramerization, dipyrromethane self-condensations in organic acid melts, Mac-Donald' sdipyrromethane " $2+2$ " condensation, Woodward' slandark dipyrromethane condensation Development in modern synthetic porphyrin chemistry was reviewed by S. Shanmugathasan and et al. ${ }^{[11]}$ The modern synthetic methods mainly divided into two ways:

i) The preparation of compounds where functionality is introduced during the formation of the porphyrin.

ii) Functionalization of performed porphyrin macrocycle.

\subsection{Adler-Longo method}

Rothemund ${ }^{[12]}$ first synthesized tetraphenyl porphyrin using benzaldehyde and pyrrole in 1936 . The reaction was carried out in a sealed tube at $150^{\circ} \mathrm{C}$ for $24 \mathrm{~h}$. The yield was low and very few substituted benzaldehydes could be used due to the drastic conditions. Improvement in this method was then

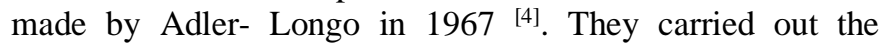
reaction of benzaldehyde and pyrrole in refluxing propionic acid for $30 \mathrm{~min}$ in open air. By using these conditions, greater variety of substitued benzaldehydes were converted into the corresponding 5, 10, 15, 20 tetraphenyl porphyrins. The reaction could also be scaled so as to give the porphyrin in multi-gram quantities. Warner ${ }^{[13]}$ under solvent free conditions reported the synthesis of tetraphenyl- porphyrin by using MW radiation.

The obvious problem in this method is the separation of mixture containing upto six different compounds. By varying the stochiometry of reagents yield of disired product can be maximised. The Adler-Longo method is often used to obtain unsymmetrically substituted tetraphenyl porphyrins with groups suitable for further modification.

While the above methods show the utility of propionic acid method, there are several drawbacks. The formation of reduced porphyrin(chlorin) invariably contaminated the product with high percentage of tarry by-products. Another problem is the failure of the reaction with aldehydes containing acid sensitive functional groups.

As our earlier work we have adopted modified Adler- Longo method to synthesized meso substituted unsymmetrical porphyrins and metalloporphyrins with two different aldehydes ${ }^{[14-18]}$.

\section{2. Lindsey method}

Many of the problems associated with the rather harsh conditions of the Adler-Longo method can be overcome using methodology developed by Lindsey et al. ${ }^{[6]}$ The Lindsey method relies on formation of porphyrinogen as an intermediate in porphyrin synthesis. The advantage of this method is that it allows the formation of product from sensitive aldehydes, in higher yields, with more facile purification. A drawback, however, is the need for higher dilution conditions i. e. reaction is not amenable to scale-up. Under acidic catalysis, an equilibrium is established with tetraphenyl porphyrinogen during the reaction of benzaldehyde and pyrrole. Once the equilibrium is established, an oxidant is added which converts porphyrinogen to the corresponding porphyrin (Scheme 2. 2 . 1). It was found using TPP as a model, that equimolar concentrations of pyrrole, benzaldehyde with boron trifluoride, at room temperature, produced optimal results. The reaction is carried out under inert conditions in dichloromethane for $1 \mathrm{~h}$, followed by addition of $2,3,5,6-$ tetrachlorobenzoquinone ( $p$-chloronil) and further hour at reflux. Lindsey and co-workers have used this method to synthesize variety of alkyl and aryl porphyrin with an average yields around $30-40 \%$, using boron trifluoride or trifluoroaceticacid as the catalyst.

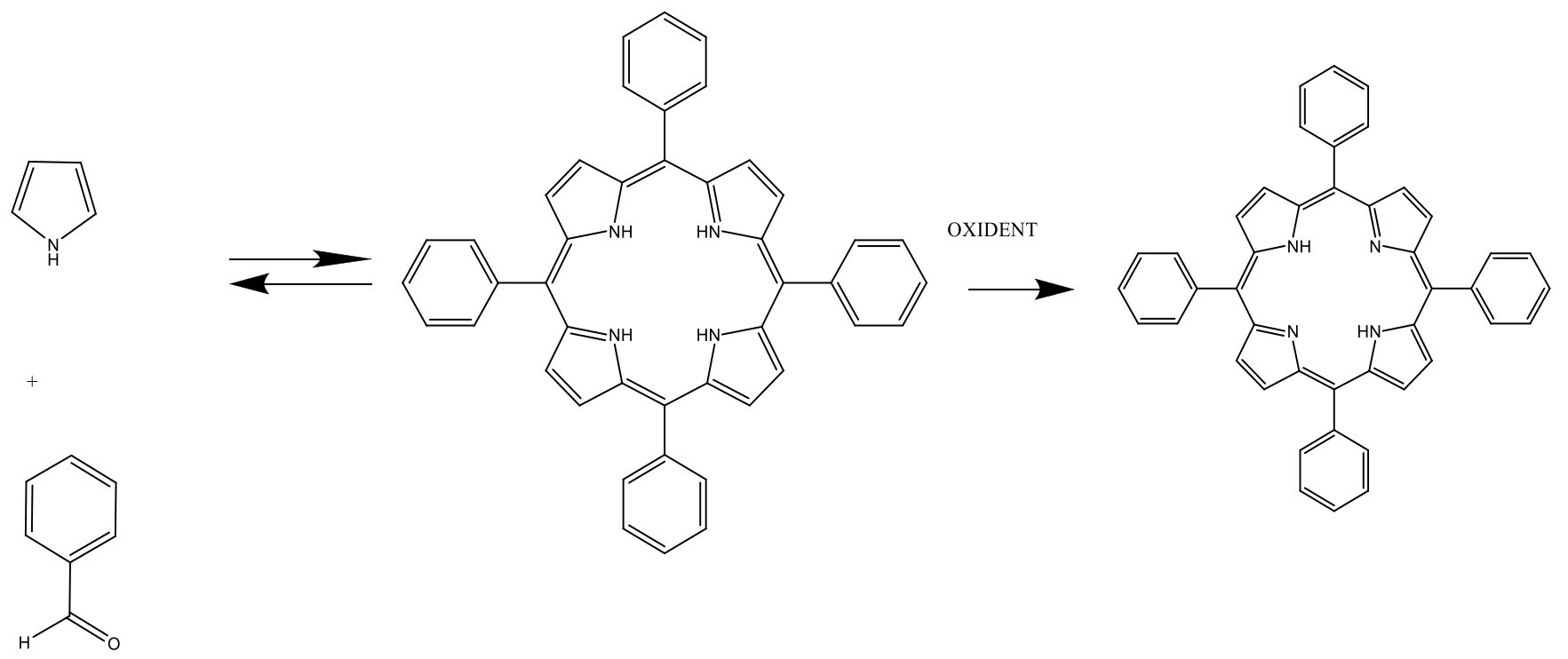

Scheme 2.2.1: Formation of Porphyrin from Porphyrinogen. 
The synthesis of sterically hindered ortho substituted tetraphenyl porphyrins described by Lindsey [5] with slight modification by the use of ethanol as a co catalyst in presence of $\mathrm{BF}_{3}$ in $30 \%$ yield.

Lindsey and coworkers ${ }^{[8]}$ reported the effect of addition of salt $\mathrm{NaC} 1$ or benzyltributylammonium chloride yield can be increased by up to two fold.

Another group Onaka [19] and Pinnavaia [20] again modified the Lindsey method by the use of clay which gave the highest yield than $\mathrm{BF}_{3}$. The synthesis of meso-teraphenylporphyrin has been reported by Warner et al. ${ }^{[13]}$ under solvent free conditions with microwave irradiation which fulfill the aspects of green chemistry.

\subsection{2+2 Porphyrin synthesis}

Porphyrins can also be prepared from dipyrromethanes by using $2+2$ synthesis. The term $2+2$ arises because the porphyrin is formed by condensation of two dipyrromethanes. Early work in this area was pioneered by MacDonald ${ }^{[9]}$ with
$2+2$ condansation. The original method involved the use of one dipyrromethane bearing two formyl groups $\propto$ to the pyrrolic nitrogen and another dipyrromethane with no $\propto-$ substitution. The scope of this methodology is that, variety of functionalized porphyrins can be synthesized in good yield with minimum isolation procedure.

Tetraarylporphyrins have also been synthesized by other modifications of $2+2$ methodology. Smith et al. ${ }^{[22]}$ have prepared meso-tetraarylporphyrins containing two-fold rotational symmetry. Smith group [21] also synthesized completely unsymmetrical tetrarylporphyrins using clay catalyzed condensation of triaryldipyrromethane with aryldipyrromethane. Lindsey and coworkers ${ }^{[23]}$ also reported the synthesis of porphyrins containing four different mesosubstituents. One of these compounds contain three different halogens attached to the phenyl rings and was prepared in 14\%, yield (Scheme-2. 3. 1).
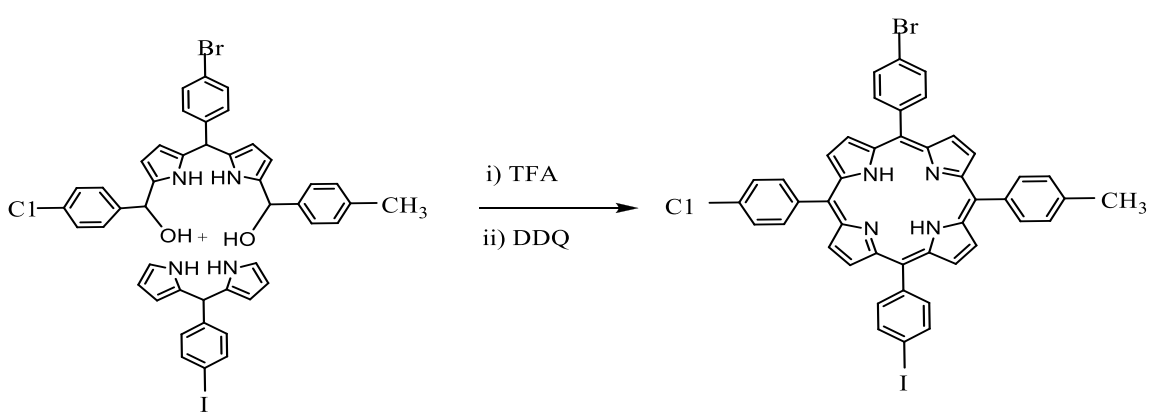

Scheme 2. 3. 1: Differentially Substituted Porphyrin.

Diphenyl- porphyrins have been synthesized by $2+2$ methodology as shown by Rose et al. ${ }^{[24]}$ by the reaction of 3 , 3'-diethyl-4, 4'-dimethyl-2, 2'-dipyrromethane with substituted benzaldehydes. They produced the 5, 15-di- $(o-$ nitrophenyl) porphyrin analogue in $45 \%$ yield.

The $2+2$ route has been very popular in recent years due to its flexibility. There are many reports of porphyrin synthesis based on this methodology. However, this methods requires suitable dipyrromethane building blocks.

In recent years, many research groups have been published the synthesis of trans- $\mathrm{AB}_{2} \mathrm{C}$ of porphyrins as a precursor for synthesis of photo induced energy transfer cascade ${ }^{[25]}$.

\subsection{3+1 Porphyrin synthesis}

The $3+1$ synthetic route involves the condensation of a tripyrrane (compound containing three pyrrole groups linked $\propto$ to the ring nitrogens by two saturated carbons) with a diformyl pyrrole. This area has seen much activity in the recent years, although this methodology has been used previously for the synthesis of expanded porphyrins and oxaand thiaporphyrins ${ }^{[26]}$.

Lash [27] described reaction of pyrrole dialdehyde and tripyrrane to prepare an octaalkylporphyrin in $60 \%$ yield. The porphyrinogen forms under acid catalysis and is then oxidised as shown in (Scheme 2. 4. 1).

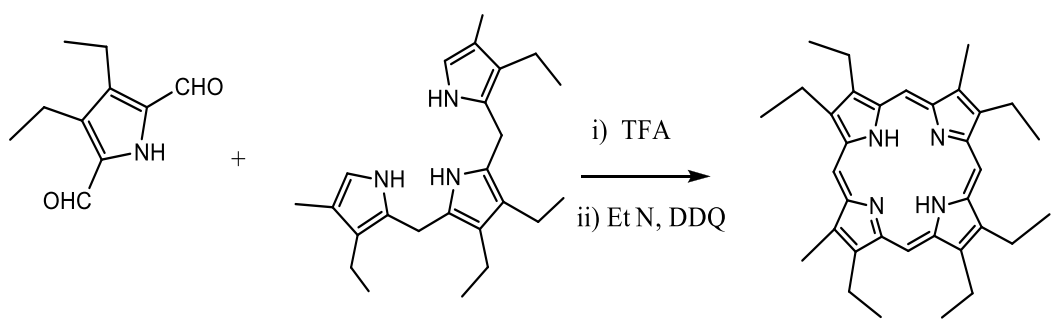

Scheme 2.4.1: Reaction conditions: (i) TFA, (ii90) $\mathrm{Et}_{3} \mathrm{~N}$, DDQ.

\section{Synthesis of functionlized porphyrins by reactions on performed porphyrins}

Reactions on the porphyrin macrocycle includes Reactions at the meso-positions, Reactions at the $\beta$-position, Cyclization reactions, Functional group interconversions, Phenyl ring transformation of aryl porphyrins.

\subsection{Reactions at the meso-position}

Formylation at the meso-position is one of the most common reaction and is carried out by the Vilsmeier formylation and the aldehyde functionality on the porphyrin can then be subjected to many conventional functional group transformations.

Halogenation of porphyrins at the meso-position is another reaction, which can give access to synthetically useful precursors. The bormo and iodoporphyrins have been used in palladium mediated coupling reactions. Therien et al. ${ }^{[28]}$ have used N-Bromo succinimide (NBS) to effect the mesodibromination of 5,15 diphenyl porphyrin, and it was reported that the halogenations ${ }^{[29]}$ took place cleanly without substitution at $\beta$-positions. 
Differentially meso-halogenated porphyrins have been prepared by Boyle et al. ${ }^{[30]}$ who meso-borminated 5, 15 diphenyl porphyrin and then iodinated. The remaining mesopositions using bis (trifluoro acetoxy) iodobenzene and iodine in quantitative yield. It was also reported that the regioselectivity of this iodinating agent was dependent on the nature of the phenyl substituent on the 5, 15-diphenyl porphyrin. [30] The halogenated e. g. mesotriiodotriarylporphyrin reported by Shultz et al. ${ }^{[31]}$ derivatives are used in the construction of butadiyne-linked porphyrin dimer.

\subsection{Functional group interconversions on the porphyrin macrocycle}

Synthetic routes based around palladium catalyzed coupling reaction have recently used in porphyrin chemistry. This is possibly due to relative ease of formation of halogenated porphyrin precursors and the vast number of substrates that can be used as coupling partners. Therien and co- workers ${ }^{[28]}$ have reported that zinc metallated meso-dibrominated 5, 15 diphenyl porphyrin to prepare various substituted porphyrins. This methodology has been extended by Boyle et al. ${ }^{[30]}$ to produce differentially substituted meso-diphenyl porphyrins. Using the differing reactivity of bromo and iodo group; the more reactive iodo group was subjected to Heck alkynylation, the bromo group was then used in a cross coupling with vinyl tributyltin under Stille conditions. More exotic molecules have been reported by Morgan et al. ${ }^{[31]}$ synthesis of barbituric acid functionalized porphyrins and chlorins, for use in photodynamic therapy.

\subsection{Phenyl ring transformations of aryl porphyrins}

Changing or introducing functionality at the phenyl rings of meso-tetraarylporphyrin or diary porphyrin is one of the most common ways to modify porphyrins. This is primarily due to fact that tetraarylporphyrins are particularly readily prepared. This approach has been used to prepare e. g. (cationic porphyrins have been synthesized from meso-tetrakis (4-

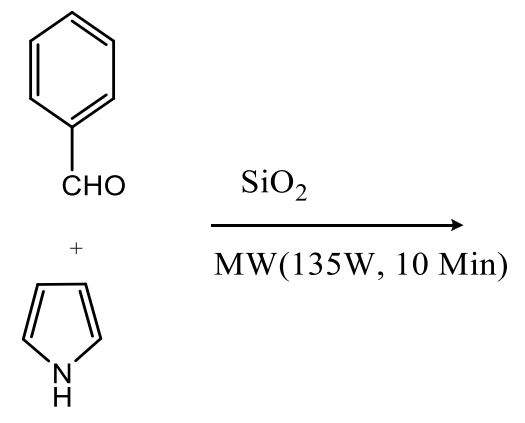

carboxymethyl) phenyl porphyrin and their interaction with DNA analysed ${ }^{[32]}$.

Regiospecific aryl nitration of TPP ${ }^{[33]}$ has been carried out to produce monoamino TPP, which was then sulfonated to produce a bifunctional porphyrin.

\section{Recent green synthetic approaches \\ 4.1. Microwave assisted reactions}

Microwave-assisted organic chemistry has grown in the last decades as a valuable and versatile tool for organic chemists. In general, compared to conventional heating methods, microwave heating has been shown to drastically reduce reaction times, increase reaction yields and enhance product selectivity, mostly reducing undesirable side reaction products. Microwave irradiation is, in our days, fully recognized as a useful tool for organic synthesis used in multi-step total synthesis, medicinal chemistry and drug discovery ${ }^{[34]}$, polymer synthesis, material science, nanotechnology and biochemical processes. The use of microwave irradiation for the synthesis and derivatization of porphyrins is reviewed by Marta Pineiro ${ }^{[35]}$ Microwaveassisted synthesis has been successfully applied for the synthesis of porphyrin macrocycles and metal insertion reactions. Microwave irradiation was also applied for the modification of the porphyrin core, synthesize chlorins and bacteriochlorins, to introduce substituents in the porphyrin periphery or to modify these substituents in order to increase the variability of functionalization.

\subsubsection{Solvent less reaction conditions}

The preparation of porphyrins under microwave (MW) activation was firstly described by Loupy and co-workers in $1992^{[36]}$. Irradiation of a mixture of pyrrole and benzaldehyde pre adsorbed on the surface of silicon dioxide for 10 minutes, using a microwave digester and open-vessel conditions, afforded 5, 10, 15, 20-tetraphenylporphyrin in $9.5 \%$ yield (Scheme 4. 1. 1. 1).

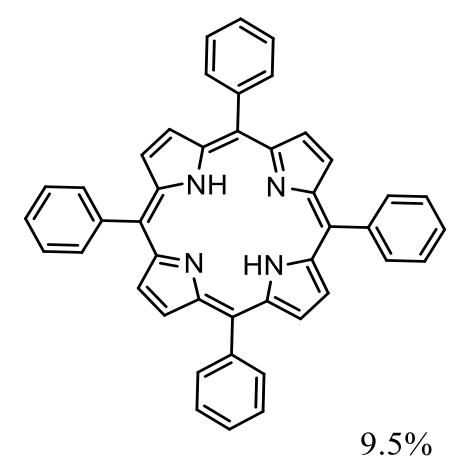

Scheme 4.1.1.1: Synthesis of tetraphenyl porphyrin

One decade after the report by Loupy this procedure was adapted for undergraduate experimental teaching [37] The research group of Raghavan [38] published a solvent-free microwave-promoted synthesis of three porphyrins in 2004. The reactions were carried out in a domestic microwave apparatus operating at $1200 \mathrm{~W}$ during 12 minutes, using HZSM-5 zeolites or Al-MCM-41 mesoporous molecular sieves as solid acidic catalysts, the latter exhibiting a better performance (Scheme 4. 1. 1. 2). 


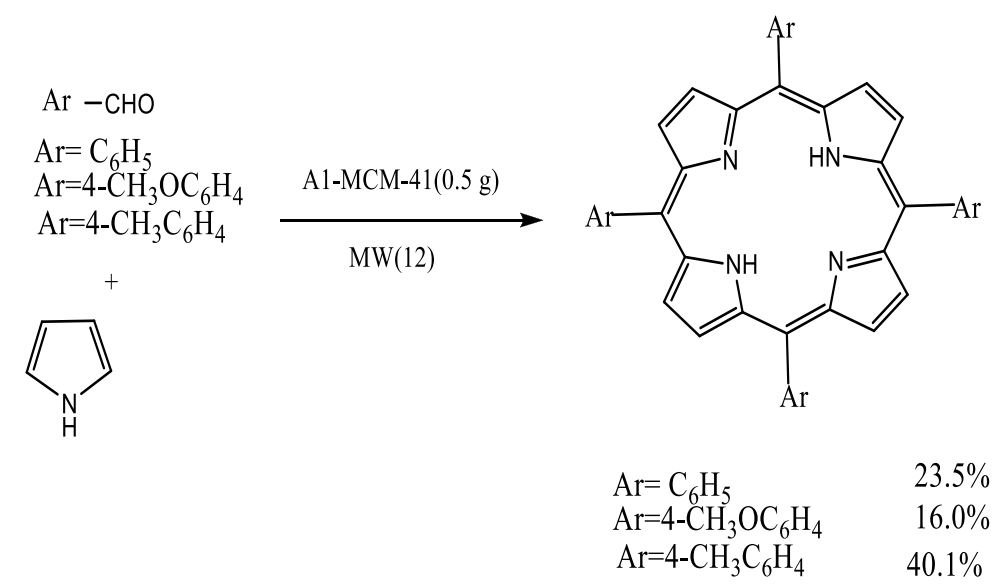

Scheme 4.1.1.2: Synthesis of symmetrical porphyrins

The 5, 10, 15, 20 tetraphenylporphinato $\mathrm{Ni}$ (II) compound was prepared, in $60 \%$ yield, in a one-pot reaction starting from pyrrole, benzaldehyde and $\mathrm{NiCl}_{2}$ ground together and poured into DBU, the mixture was stirred for 10-15 minutes and irradiated in a microwave domestic oven at $500 \mathrm{~W}$ in a silica gel bath for 8 minutes with two-minute intervals in between the reaction time ${ }^{[39]}$.

\subsubsection{Synthesis in solution}

Chauhan and co-workers [40] reported in 2001, the condensation of equimolar amounts of a series of aryl aldehydes and pyrrole in an open vessel, employing propionic acid as solvent and making use of a microwave domestic oven in an adaptation of the classical Adler method. Although the authors used a domestic oven (power not disclosed) microwave irradiation for 3 to 5 minutes, followed by cooling to room temperature, and further purification, the target mesosubstituted porphyrins and were obtained with poor to moderate isolated yields. Nevertheless, comparing microwave irradiation using $5 \mathrm{~mL}$ of propionic acid with conventional heating (C. H.) using $160 \mathrm{~mL}$ of propionic acid, all porphyrins were obtained with higher isolated yields under microwave irradiation, Recently, Mikus and co-workers ${ }^{[41]}$ reviewed this method in an attempt to optimize the microwave-assisted synthesis of mesotetraphenylporphyrin.

$\mathrm{Hu}$ and co-workers ${ }^{[42]}$ used the method described by Chauman for the synthesis of 5, 10, 15, 20-tetrakis(4tbutylphenyl) porphyrin in $56 \%$ yield. This porphyrin was transformed into the corresponding $\mathrm{Mg}(\mathrm{II}), \mathrm{Zn}(\mathrm{II}), \mathrm{Cu}(\mathrm{II})$, In(III) and $\mathrm{Al}(\mathrm{III})$ complexes under microwave irradiation using DBU as catalyst in high yields, (Scheme 4. 1. 2. 1).

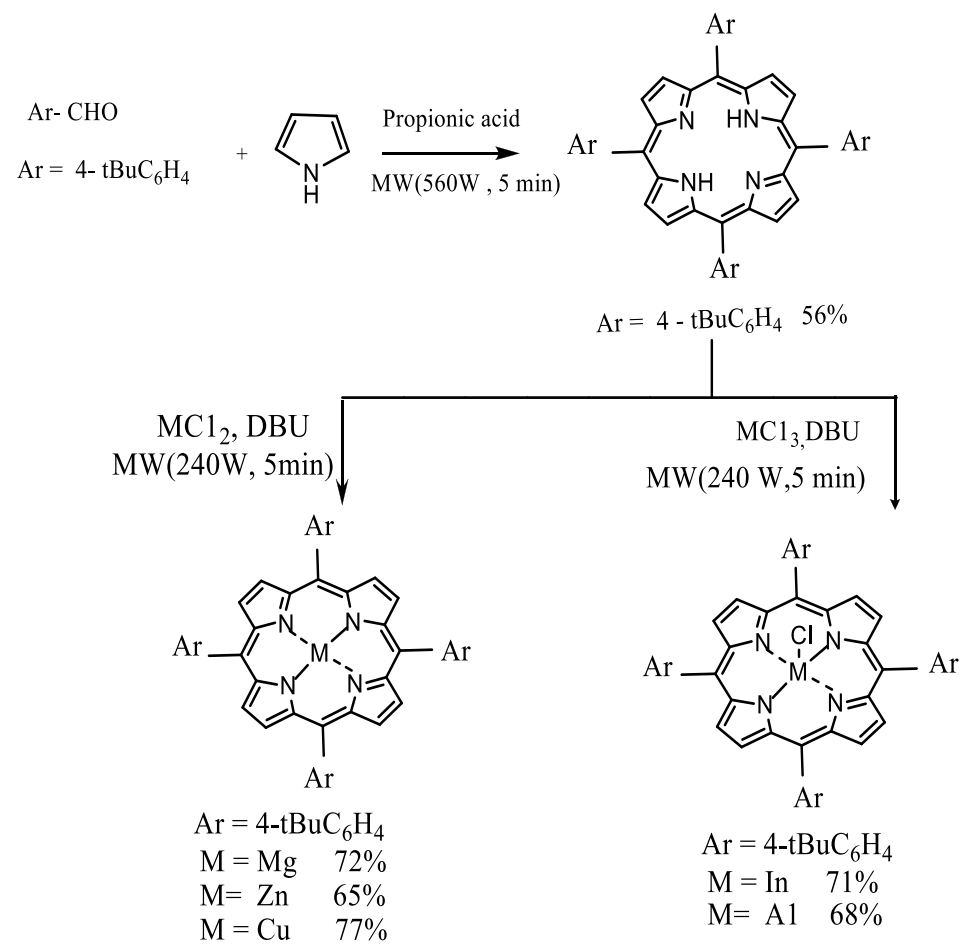

Scheme 4.1.2.1: Synthesis of symmetrical porphyrin and matalloporphyrins in solution

\subsection{Functionalization through organometallic techniques} The employment of organometallic reactions has become common in porphyrin synthesis. Palladium-catalyzed crosscoupling reactions are now standard techniques for constructing carbon-carbon bonds in porphyrin synthesis. In addition, iridium- or palladium-catalyzed direct $\mathrm{C}-\mathrm{H}$ functionalization of porphyrins is emerging as an efficient way to install various substituents onto porphyrins. Furthermore, the copper mediated Huisgen cycloaddition reaction has become a frequent strategy to incorporate 
porphyrin units into functional molecules. The use of these organometallic techniques, along with the traditional porphyrin synthesis, now allows chemists to construct a wide range of highly elaborated and complex porphyrin architectures.

Porphyrin is one of the archetypal functional molecules, playing an important role in diverse areas of scientific research owing to its unique electronic and optical properties. To achieve these fascinating functions, design and synthesis of structurally diverse porphyrin molecules is essential. For example, hydrophilic substituents are often installed to increase solubility in aqueous media and enhance membrane permeability for cancer therapy applications. Porphyrins for solar cell applications require donor and acceptor moieties at certain positions. For energy and electron transfer studies, porphyrin oligomers with adequate potential gradients have to be prepared. These complex and elaborate structures are difficult to prepare using only conventional porphyrin synthesis. Undoubtedly, stoichiometric organometallic reagents and catalytic transition metal complexes are powerful tools in organic synthesis. Transition metal catalysis not only enables high selectivity, high efficiency, and environmentally benign processes but also new types of direct transformations to short-cut lengthy multistep syntheses, which are not attainable by conventional methodologies.

The use of palladium-catalyzed cross coupling reactions in porphyrin synthesis expands every year. Palladium-catalyzed coupling techniques include Suzuki- Miyaura coupling, Migita-Kosugi-Stille coupling, Negishi coupling, Sonogashira coupling, the Mizoroki- Heck reaction, and Buchwald-Hartwig amination. These methodologies have been successfully applied to derivatize halogenated porphyrins at various positions of the macrocyclic core and peripheral substituents. Copper-mediated transformations have a long history and have been important tools in organic synthesis. These methodologies include Glaser-type dimerization of terminal alkynes and the Ullmann coupling reaction, which are also useful in the synthesis of butadiynelinked porphyrins and heteroatom-substituted porphyrins. However, the most useful and most frequently employed copper-catalyzed reaction in porphyrin synthesis is clearly the Huisgen cycloaddition reaction between organic azides and terminal alkynes, the so called "click reaction" or coppercatalyzed azide-alkyne cycloaddition ${ }^{[43]}$. This methodology is a powerful and general way to connect two units into one molecule in a highly efficient manner.

Post functionalization of porphyrins can be further classified into two categories, one is core-functionalization and the other is peripheral-functionalization. In the case of corefunctionalization, the core-skeleton of the porphyrin macrocycle is directly functionalized at the meso- and/or $\beta$ positions. On the other hand, functional groups can be introduced on the peripheral substituents. The most of these functionalizations are performed using various types of porphyrins bearing reactive substituents such as halogens, alkynes, and metals on the porphyrin core or the substituents. Organolithium reagents work as effective nucleophiles to attack at meso- and $\beta$-positions of porphyrins, enabling direct functionalization of porphyrins ${ }^{[44]}$.

Iridium-catalyzed direct $\mathrm{C}-\mathrm{H}$ borylation was developed by Smith [45], Hartwig, Ishiyama, and Miyaura and this methodology has been employed for post functionalization to modify relatively simple and unfunctionalized porphyrins. Palladium-catalyzed $\mathrm{C}-\mathrm{H}$ arylation of porphyrins is also emerging as a direct method to install various aromatic substituents onto porphyrins

Stepwise addition and oxidation reactions of with two different lithium reagents furnished $\mathrm{A}_{2} \mathrm{BC}$-type porphyrin having three different groups at the meso-positions (Scheme 4. 2. 1) ${ }^{[46]}$.
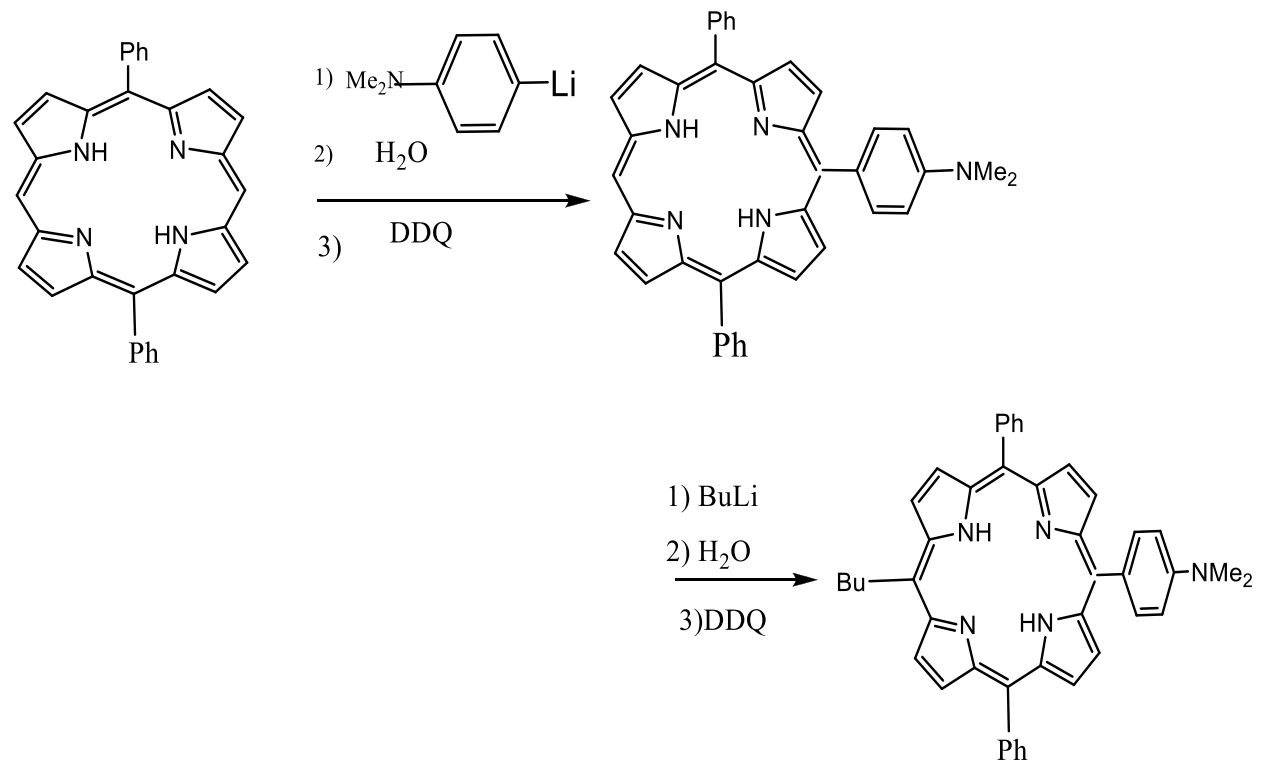

Scheme 4.2.1: Sequential functinalization of 5, 15 diphenylporphyrin

The sequential methodology using organolithium reagents can be further expanded to the direct introduction of useful functionality. A dithianyl group serves as an acyl anion equivalent. The reaction of 5,15 diphenylporphyrin with dithianyllithium, DDQ and $\mathrm{BF}_{3}, \mathrm{OEt}_{2}$ efficiently converted to formyl porphyrin after deprotection ${ }^{[47-48]}$.
Senge and co-worker reported the synthesis of meso-mesolinked diporphyrins on the basis of the addition reaction of organolithium reagents (Scheme 4. 2. 2). ${ }^{[49-50]}$ The post functionalization of porphyrins and related compounds through catalytic and stoichiometric organometallic methodologies has been reviewed by Hiroshi Shinokubo et al. [51] 

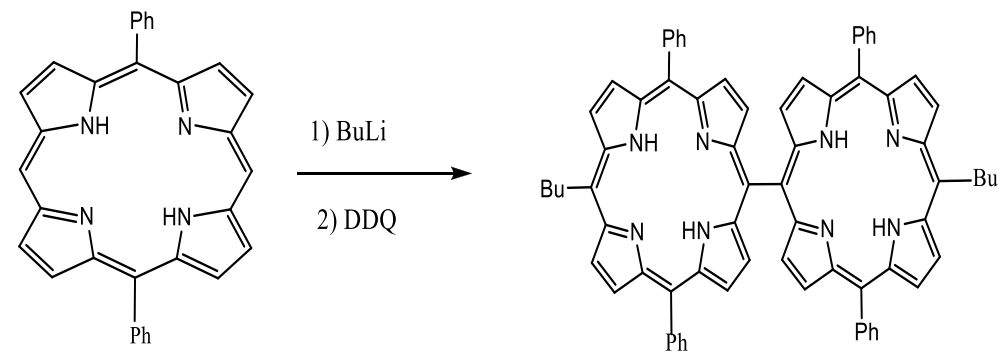

Scheme 4.2.2: Synthesis of diporphyrin

\subsection{Porphyrin macrocycle modifications}

Pyrrole ring contracted or expanded porphyrin ide in recent years, $\beta$ pyrrolic positions leading to pyrrole modified porphyrins containing four, five, six and seven heterocycles. azeteoporphyrins, porpholactonesand morpholino porphyrins are representative examples of porphyrin ides.

Tome AC and coworkers ${ }^{[52]}$ have reported the methods of modification of porphyrin macrocycle and the potential applications of resulting porphyrin ides.
Such compounds have been prepared by (i) the structural modification of already existing porphyrins via, for instance, cycloaddition reactions, electrophillic or nucleophillic aromatic substitutions, pyrrole ring contraction or expansion reactions or (ii) by constraction of porphyrin macrocycle using pyrollic building blocks and also $3+1$ method.

Paney and coworkers reported a versatile to monody (2- oxo pyri) porphyrins ${ }^{[53]}$ (Scheme 4. 3. 1).

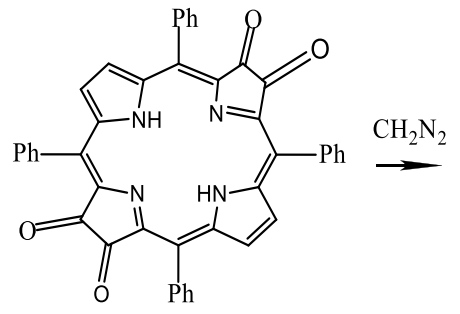

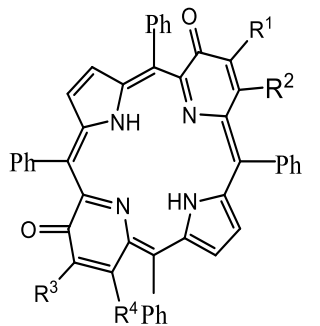

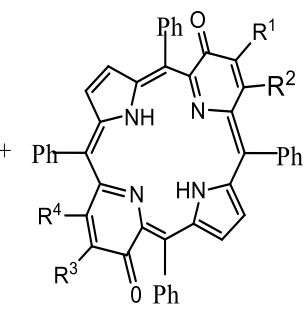

$$
\begin{aligned}
& \mathrm{R}^{1}=\mathrm{R}^{3}=\mathrm{OMe}, \mathrm{R}^{2}=\mathrm{R}^{4}=\mathrm{H} \\
& \mathrm{R}^{1}=\mathrm{R}^{4}=\mathrm{OMe}, \mathrm{R}^{2}=\mathrm{R}^{3}=\mathrm{H} \\
& \mathrm{R}^{2}=\mathrm{R}^{4}=\mathrm{OMe}, \mathrm{R}^{1}=\mathrm{R}^{3}=\mathrm{H}
\end{aligned}
$$

Scheme 4.3.1: Synthesis of di (2- Oxopyri) porphyrins ${ }^{[53]}$

\subsection{Synthesis by using acid catalyst}

4. 4. 1: Synthesis by using zeolite

The cost-efficient method for unsymmetrical meso aryl porphyrins using NaY zeolite as an inorganic acid catalyst ${ }^{[54]}$ (Scheme 4.4.1.1).
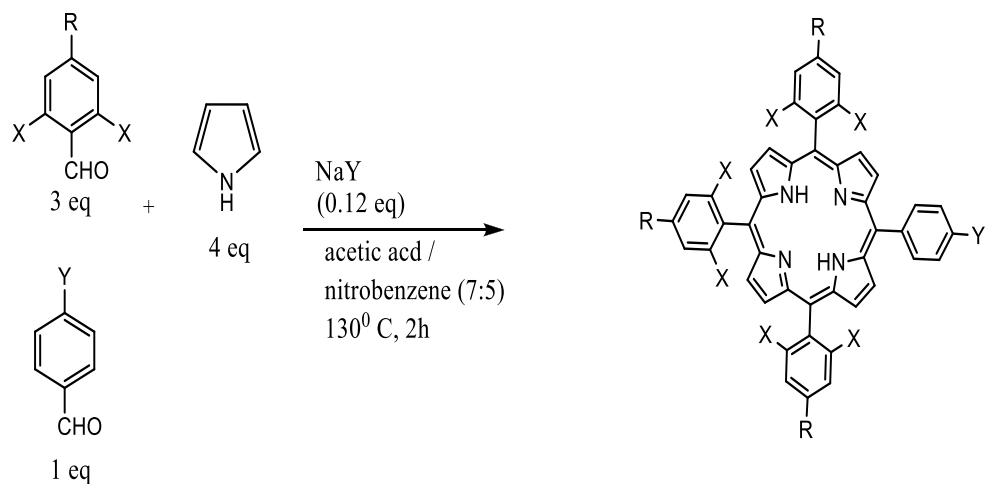

$1: \mathrm{X}=\mathrm{H} ; \mathrm{Y}=\mathrm{OH} ; \mathrm{R}=\mathrm{H}(16 \%)$

$2: \mathrm{X}=\mathrm{H} ; \mathrm{Y}=\mathrm{OH} ; \mathrm{R}=\mathrm{O}-(2-$ Ethylhexyl) $(15 \%)$

$3: \mathrm{X}=\mathrm{H} ; \mathrm{Y}=\mathrm{COOH} ; \mathrm{R}=\mathrm{H}(17 \%)$

$4: \mathrm{X}=\mathrm{F} ; \mathrm{Y}=\mathrm{COOH} ; \mathrm{R}=\mathrm{H}(13 \%)$

5: $\mathrm{X}=\mathrm{F} ; \mathrm{Y}=\mathrm{NHAc} ; \mathrm{R}=\mathrm{H}(17 \%)$

$6: \mathrm{X}=\mathrm{Cl} ; \mathrm{Y}=\mathrm{NHAc} ; \mathrm{R}=\mathrm{H}(13 \%)$

Scheme 4.4.1.1: Synthesis of unsymmetrical porphyrins

\subsection{2: Synthesis by using ionic liquids}

In Lindsey method, condensation of benzaldehyde with pyrrole in halogenated solvent at room temperature followed by oxidation effectively produced TPP and in Adler method, refluxing propionic acd containing benzaldehyde and pyrrole open to air followed by filtration of precipitated TPP obtained in $20 \%$ yield. 
However, the advantage of ionic liquid methodology is not required the oxidant and halogenated solvent unlike Lindsey method. Ionic liquids could be suitable and environmentally safer replacements for the volatile, toxic, and flammable organic solvents. another advantage of ionic liquid is that it is reusable without loss of its catalytic activity.

Novel synthetic methodology for metalloporphyrins using ionic liquid has been reported by Sing Ram ${ }^{[55]}$ (Scheme 4. 4. 2. 1).

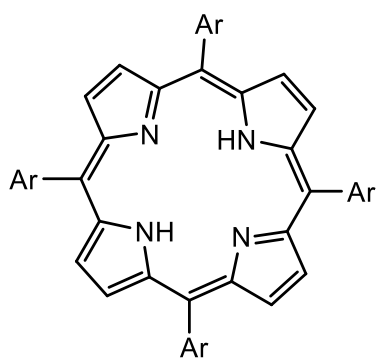

\section{$\mathrm{MX} 2 /[\mathrm{hmim}][\mathrm{Br}]$}

r.t.

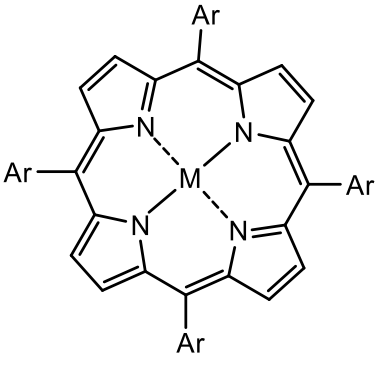

3. $\mathrm{Ar}=$<smiles>Cc1c(F)cccc1Cl</smiles>

5. $\mathrm{Ar}=$<smiles>Fc1c(F)c(F)c(F)c(F)c1F</smiles>

Scheme 4.4.2.1: Synthesis of symmetrical metalloporphyrins

Satoshi Kitaoka and et al. ${ }^{[56]}$ reported the effect of acidity on different ionic liquids in synthesis of tetrapyridyl porphyrin using Adler method (Scheme 4. 4. 2. 2)
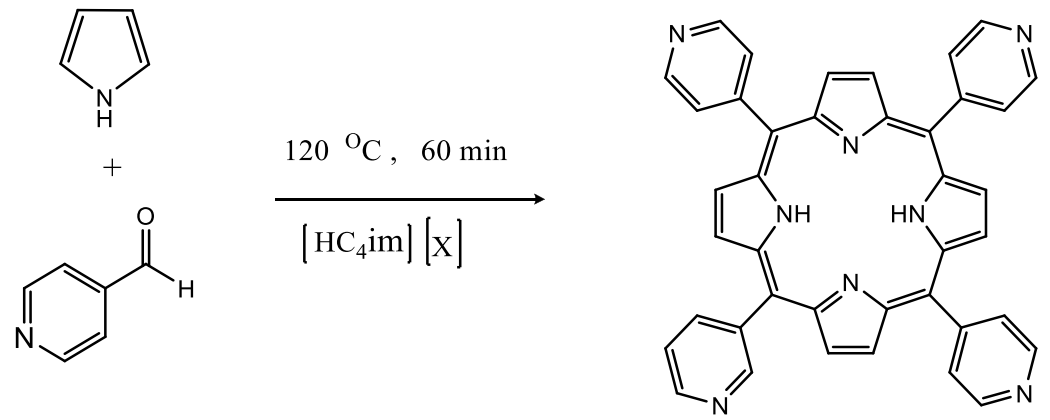

Anion of ionic liquid, $\mathrm{X}=\mathrm{CF}_{3} \mathrm{SO}_{3}^{-}, \mathrm{ClO}_{4}^{-}, \mathrm{C1}^{-}, \mathrm{CF}_{3} \mathrm{CO}_{2}^{-} \mathrm{BF}_{4}^{-}$

Scheme 4.4.2.2: Synthesis of tetrapyridyl porphyrin

\subsection{3: Mechanochemical synthesis}

Mechanochemical chemistry, where chemical reactions are promoted by grinding reagents together either using a mortar and pestle or automated ball mill, is an offen overlooked approach to molecular synthesis that is currently gaining ground due to the relative simplicity of reaction condition, potential for elimination of solvent. Applicability of this approach for organic reactions, especially carbon -carbon bond forming reactions is still emerging area.

Hamilton TD [57] and coworkers have described machanochemical synthesis of series meso substituted porphyrins involving the grinding of benzaldehyde and pyrrole in presence of acid catalyst to give a solid pink powder. This powder can then be oxidized either in air, in chloroform with an organic oxidizer, or in second grinding step with an oxidizing agent to produce TPP and this approach has been also extended for various substituted porphyrins. (Scheme 4. 4. 3. 1). Two green chemistry approach, E factor and Eco scale Show that, the mechanochemical process is more efficient from an environmental perspective. 


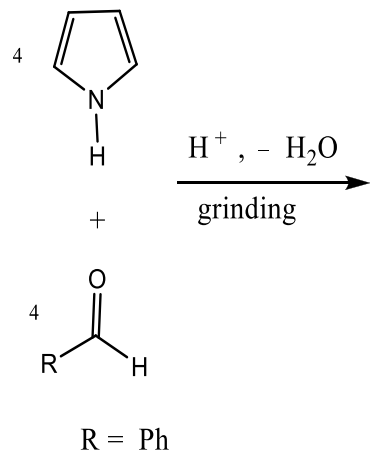

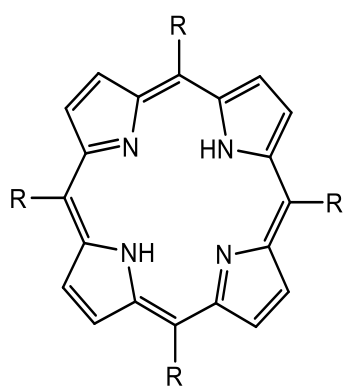

pink powder containing porphyrinogen and other reduced porphyrin intermediates

Scheme 4.4.3.1: Two step Synthesis of porphyrin involving mechanochemistry.

\section{Conclusion}

The various structural modifications around the microcyclic ring of porphyrins and metalloporphyrins evaluate are for their usefulness in treating various disease conditions. These molecules being a central body of pharmacophore, holds different types of substituents. Based on their various physiochemical properties, they exerted a diversified range of therapeutic efficacy. Thus we can conclude that this review will definitely provide the researchers with through understanding of the structural activity relationship study, which further helps in designing good large number of porphyrins and metalloporphyrins and their analogue compounds with strong impact in curing many fatal disorders and also contributes to motivate other researchers to embrace this subject.

\section{References}

1. (a) Gao J, Gu H, Xu B. Acc. Chem. Res. 2009; 42:10971107.

(b) Li WS, Aida T. Chem. Rev. 2009; 109:6047-6076 and references therein.

2. Drain CM, Varotto A Radivojevic I. Chem. Rev. 2009; 109:1630-1658.

3. (a) Rothemund PJ. Am. Chem. Soc. 1935; 57:2010-2011.

(b) Rothemund P, Menotti AR J. Am. Chem. Soc. 1941; 63:267-270.

4. (a) Adler AD, Longo FR, Shergalis WJ. Am. Chem. Soc. 1964; 86:3145-3149.

(b) Adler AD, Longo FR, Finarelli JD, Goldmacher J, Assou J, Korsakoff LJ. Org. Chem. 1967; 32:476.

5. Lindsey JS, Wagner RW. J. Org. Chem. 1989; 54:828836.

6. (a) Lindsey JS, Hsu HC, Schreiman IC. Tetrahedron Lett. 1986; 27:4969-4970.

(b) Lindsey JS, Schreiman IC, Hsu C, Kearney PC, Marguerettaz AM. J. Org. Chem. 1987; 52:827-836.

7. Lindsey JS, MacCrum KA, Tyhonas JS, Chuang YY. J. Org. Chem. 1994; 59:579-587.

8. Li F, Yang K, Tyhonas JS, MacCrum KA, Lindsey JS. Tetrahedron. 1997; 53:12339-12360.

9. Arsenault GP, Bullock E, MacDonald SF. J. Am. Chem. Soc. 1960; 82:4384-4389.

10. (a) Lindsey JL. Acc. Chem. Res. 2010; 43:300-311 and references therein.

(b) Boyle NM, Rochford J, Pryce MT. Coord. Chem. Rev. 2010; 254: 77-102 and references therein.

(c) Burrell AK, Officer DL, Plieger PG, Reid D. C W. Chem. Rev. 2001; 101:2751-2796 and references therein.
11. (a) Wijesekera TP, Dolphin D. In Metalloporphyrin in Catalytics Oxidations, Sheldon RA, Ed., Marcel Dekker, New York, 1994, 231p.

(b) Review by S. Shanmugathasan S, Edwards C, Boyle RW. Tetrahedron. 2000; 56:1025.

12. Rothemund PJ. Am. Chem. Soc. 1936; 58:625.

13. Marvin GW, Gary LS, James EH. Green Chemistry. 2001; 3(6):267.

14. Bandgar BP, Gujarathi PB. Synthesis and characterization of new meso substituted unsymmectrical metalloporphyrins, J. Chem. Sci. 2008; 120(2):1-8.

15. Bandgar BP, Gujarathi PB. Synthesis of new meso substituted unsymmectrical porphyrins, Indian Journal of Heterocyclic Chemistry. 2008; 17:249-252.

16. Gujarathi PB Preparation and Spectral Studies of Cobalt (II), Nickel(II), Copper (II) and Zinc(II) Complexes of New meso Substituted Unsymmectrical Porphyrins International Journal of Chemical and Pharmaceutical Sciences. 2016; 7:3.

17. Gujarathi PB. Synthesis and Characterization of Some Novel meso functionlized Unsymmectrical metalloporphyrins, Chemical Science Transactions. 2016; $5: 4$.

18. Gujarathi PB. New meso Substituted Unsymmectrical Porphyrins: Synthesis and Spectral Studies, Acta Chimica Pharmaceutica Indica. 2016; 6(3):104-110.

19. Onaka M, Shinodo T, Izumi Y, Nolen E. Chem. Lett, 1993, 117.

20. Cady SS, Pinnavaia T, Inorg J. Chem. 1978; 17:1501.

21. Wallace DM, Leung SM, Senge MO, Smith KM. J. Org. Chem. 1993; 58:7245.

22. Wallace DM, Smith KM. Tetrahedron Lett. 1990; 31:7265.

23. Lee CH, Li F, Iwamoto K, Dadok J, Brothner By AA, Lindsey JS. Tetrahedron. 1995; 51:11645.

24. Lecas - Nawrocka A, Boitrel B, Rose E, Tetrahedron Lett. 1992; 33:481.

25. Miller MA, Lammi RK, Prathapan S, Holten D, Lindsey JS. J. Org. Chem. 2000; 65:6634.

26. Broadhurst MJ, Grigg R, Johnson AW. J. Chem. Soc (c), 1971, 3681.

27. Lash TD. J. Porphyrins Phthalocyanines. 1997; 1:29.

28. Dimagno SG, Lin VSY, Therien M. J. Org. Chem. 1993; 58:5983.

29. Blake IM, Anderson HL, Beljonne D, Bredas JL, Clegg WJ. Am. Chem. Soc. 1998; 120:10764.

30. Shanmugathasan S, Johnson CK, Dolphin D, Edwrds C, Boyle RW, Proceeding of Seventh Biennial congress of 
the International Photodynamic Association, Nantes, France, 1998, 133.

31. Robinson BC, Morgan AR. Tetrahedron Lett. 1993; 34:3711.

32. Robic N, Bied-Charreton C, Fauvet MP, Beaur CV, Salmon L, Gaudemer A. Tetrahedron Lett. 1990; 31:4739.

33. Jr. Kruper WJ, Chamberlain TA, Kochanny M. J. Org. Chem. 1989; 54:2753.

34. (a) Chighine A, Sechi G, Bradley M. Tools for efficient high-throughput synthesis. Drug. Discov. Today. 2007; 12:459-464.

(b) Alcazar J, Diels G, Schoentjes B. Microwave Assisted Medicinal Chemistry Mini-Rev. Med. Chem. 2007; 7:345-369;

(c) Kappe CO, Dallinger D. The impact of microwave synthesis on drug discovery. Nat. Rev. Drug. Discovery. 2006; 5:51-68.

35. Marta Pineiro Microwave-assisted synthesis and reactivity of porphyrins Current Organic Synthesis. 2014; 11:89-109.

36. Petit A, Loupy A, MaiUard P, Momenteau M. Microwave irradiation in dry media: A new and easy method for synthesis of tetrapyrrolic compounds. Syn. Commun. 1992; 22:1137-1142.

37. Kishan MR, Rani VR, Devi PS, Kulkarni SJ, Raghavan $\mathrm{KV}$. Anovel zeolite based stationary phases for in situ synthesis and evaluation of porphyrins and calix pyrroles. J. Mol. Catal. A: Chem., 2007; 269:30-34.

38. Kishan MR, Rani VR, Murty MR VS, Devi PS, Kulkarni SJ, Raghavan KV. Synthesis of calixpyrroles and porphyrins over molecular sieve catalysts. J. Mol. Catal. A: Chem. 2004; 223:263-267.

39. Sharma RK, Ahuja G, Sidhwani IT. A new one pot and solvent-free synthesis of nickel porphyrin complex. Green Chem. Lett. Rev. 2009; 2:101-105.

40. Chauhan SMS, Sahoo BB, Srinivas KA. Microwaveassisted synthesis of $5,10,15,20$ - tetraarylporphyrins. Synth. Commun. 2001; 31:33-37.

41. Mikus A, Bielinska ME, Lipinska T, Ostrowski S. Synthesis of meso- TPP revisited: its "green oriented" optimization under controlled microwave heating. Synth. Commun. 2011; 41:3703-3713.

42. Liu MO, Tai C-H, Wang W-Y, Chen J-R, Hu AT, Wei TH. Microwave-assisted synthesis and reverse saturable absorption of phthalocyanines and porphyrins. J. Organomet. Chem. 2004; 689:1078-1084.

43. Huisgen R. 1, 3-Dipolar Cycloadditions. Past and Future. Angew. Chem., Int. Ed. 1963; 2:565-598.

44. Senge MO. Nucleophilic Substitution as a Tool for the Synthesis of Unsymmetrical Porphyrins. Acc. Chem. Res. 2005; 38:733-743.

45. Cho JY, Tse MK, Holmes D, Maleczka RE, Smith MR. Remarkably Selective Iridium Catalysts for the Elaboration of Aromatic C-H Bonds. Science. 2002; 295:305-308.

46. Feng X, Senge MO. An Efficient Synthesis of Highly Functionalized Asymmetric Porphyrins with Organilithium Reagents. J. Chem. Soc., Perkin Trans. 2001; 1:1030-1038.

47. Senge MO, Hatscher SS, Wiehe A, Dahms K, Kelling A. The Dithianyl Group as a Synthon in Porphyrin Chemistry: Condensation Reactions and Preparation of Formylporphyrins under Basic Conditions. J. Am. Chem. Soc. 2004; 126:13634-13635.
48. Dahms K, Senge MO, Bakar MB. Exploration of mesoSubstituted Formylporphyrins and Their Grignard and Wittig Reactions. Eur. J. Org. Chem, 2007, 3833-3848.

49. Feng X., Senge MO. Regioselective Reaction of 5, 15 Disubstituted Porphyrins with Organolithium Reagents-Synthetic Access to 5, 10, 15-Trisubstituted Porphyrins and Directly meso-meso- Linked Bisporphyrins. J. Chem. Soc., Perkin Trans. 2000; 1:3615-3621.

50. Senge MO, Feng X. Synthesis of Directly meso-meso Linked Bisporphyrins Using Organolithium Reagents. Tetrahedron Lett. 1999; 40:4165-4168.

51. Satoru Hiroto, Yoshihiro Miyake, and Hiroshi Shinokubo, Synthesis and Functionalization of Porphyrins through Organometallic Methodologies Chem. Rev. 2017; 117:2910-3043.

52. Costa LD, Costa JIT, Tome AC. Porphyrin macrocycle modification: pyrrole ring contracted or - expanded porphyrinoids. Molecules. 2016; 21:320.

53. kozyrev AN, Alderfer JL, Dougherty TJ, Pandey RK. Synthesis of mono- and di (oxopyri) porphyrins:anew approach through ring enlargement with dianzomethane Angew. Chem. Int. Ed. 1999; 38:126-128.

54. Calvete MJF, Dias LD, Henriqes CA, Pinto SMA, Carrilho RMB, Pereira MM. A cost-efficient method for unsymmectrical meso aryl porphyrin synthesis using NaY Zeolite as an inorganic acd catalyst, Molecule. 2017; 22:741.

55. Sing Ram, Geetanjali Novel synthetic method for metalloporphyrins in ionic liquid J. Braz. Chem. Soc. 2005; 16(3B):666-668.

56. Kitaoka Satoshi, Nobuoka kaoru, Ihara Keita, ishikawa Yuichi A simple method for efficient synthesis of tetrapyridyl porphyrin using Adler method in acidic ionic liquids.

57. Hannah Shy, Paula Mackin, Andrea S. Orvieto, Deepa Gharbharan, Geneva R. Peterson, Nick Bampos and Tamara D. Hamilton. Faraday Discuss. 2014; 19(170):5969. 